'Unidad de Arritmias y

Laboratorio de Electrofisiología

Cardíaca, Hospital Militar de

Santiago. Santiago, Chile.

${ }^{2}$ Servicio de Infectologia, Hospital

Militar de Santiago. Santiago,

Chile.

${ }^{3}$ Unidad Coronaria, Hospital

Militar de Santiago. Santiago,

Chile.

Los autores declaran no tener conflictos de interés.

Trabajo no recibió financiamiento.

Recibido el 8 de mayo de 2020, aceptado el 21 de agosto de 2020.

Correspondencia a: Dr. José Pardo G.

Unidad de Arritmias y Laboratorio de Electrofisiología Cardíaca. Hospital Militar de Santiago. Av. Fernando Castillo Velasco 9100, La Reina. Santiago, Chile. joseapardo@hotmail.com

\section{Patrón electrocardiográfico Brugada tipo I asociado a un cuadro febril por Influenza B. A propósito de la pandemia de COVID-19. Caso clínico}

\author{
JOSÉ PARDO ${ }^{1}$, ALBERTO FICA $^{2}$, ANDRÉ CARTES ${ }^{2, a}$, \\ GONZALO LIRA ${ }^{1}$, JORGE TORRES ${ }^{3}$
}

\section{Type I Brugada electrocardiographic pattern associated with Influenza B and fever. Report of a case}

We report a 44-year-old male who was admitted for Influenza $B$ and fever, presenting a type I Brugada pattern on the electrocardiogram. He evolved without cardiovascular symptoms. The pharmacological test with intravenous Procainamide reproduced type I Brugada pattern and the programmed electrical stimulation was negative for ventricular arrhythmias. He was discharged without incidents. Clinical aspects of Brugada syndrome and the importance of fever are discussed in the current context of the COVID-19 pandemic.

(Rev Med Chile 2020; 148: 1368-1370)

Key words: Brugada Syndrome; COVID-19; Fever; Influenza, Human.
$\mathrm{E}$ 1 síndrome de Brugada ( $\mathrm{SBr}$ ), descrito en 1992, se caracteriza al electrocardiograma (ECG) por una imagen de bloqueo de rama derecha y elevación del segmento ST en derivaciones precordiales derechas, asociado a arritmias ventriculares malignas y muerte súbita (MS) en ausencia de cardiopatía estructural ${ }^{1}$.

El SBr es una canalopatía de transmisión autosómica dominante con múltiples mutaciones genéticas que afectan al gen SCN5A localizado en el cromosoma 3 y que sintetiza los canales del sodio. ${ }^{2}$

En el ECG, el patrón Brugada (Br) tipo I se observa con elevación descendente del segmento $\mathrm{ST} \geq 2 \mathrm{~mm}$ seguida de onda T negativa de V1 a V3 y es el único patrón típico que permite el diagnóstico definitivo de $\mathrm{SBr}^{3}$.

Un cuadro febril de origen infeccioso puede hacer evidente un patrón Br tipo I al ECG y desenmascarar un SBr hasta ese momento desconocido ${ }^{1-4}$.

Comunicamos el caso de un paciente conocido en el año 2013, que se presentó con un ECG con patrón Br tipo I en el curso de una influenza B y fiebre.

Este reporte puede contribuir al manejo actual de pacientes con patrón Br tipo I o SBr que pudiesen presentarse en época de pandemia por COVID-19. Se obtuvo consentimiento informado para su publicación.

\section{Historia Clínica}

Ingresó a Urgencia un hombre de 44 años por un cuadro de 2 días de evolución de odinofagia, tos seca, cefalea, mialgias intensas y sensación febril. El ECG, con $38,7^{\circ} \mathrm{C}$, mostró un patrón $\mathrm{Br}$ tipo I (Figura 1A). Al examen físico destacó una faringe congestiva y taquicardia de 120 latidos por minuto. Se hospitalizó en Unidad Coronaria donde la monitorización ECG mostró desaparición del patrón Br tipo I al bajar la temperatura (Figura 1B). 
Sin antecedentes familiares de MS. El estudio con inmunofluorescencia para virus respiratorio resultó positivo para influenza B y la radiografía de tórax mostró refuerzo intersticial perihiliar. La ecocardiografía 2D fue normal. Se trató con paracetamol y oseltamivir oral y evolucionó de manera satisfactoria sin complicaciones. Previo al alta se realizó un test de provocación farmacológica con procainamida endovenosa, agente bloqueador de canal de sodio, que reprodujo el patrón $\mathrm{Br}$ tipo I (Figura 1C). El estudio electrofisiológico con estimulación eléctrica programada en ventrículo derecho resultó normal, sin inducción de arritmias ventriculares. El paciente se dio de alta en buenas condiciones, sin antiarrítmicos, advirtiéndosele de la importancia de tratar agresivamente cualquier alza febril y evitar drogas contraindicadas en el SBr, señaladas en el sitio web (www.brugadadrugs.org).

\section{Discusión}

El SBr representa 4 a $12 \%$ de todas las causas de MS y $20 \%$ de las que ocurren sin cardiopatía estructural ${ }^{2}$.

Su prevalencia en Europa se estima entre 1-5/10.000 habitantes y en el sudeste asiático 12/10.000 habitantes ${ }^{3}$.

El paciente puede ser asintomático o presentarse con síncope, fibrilación auricular paroxística o bloqueo aurículo-ventricular, siendo lo más dramático la MS por taquicardia ventricular polimorfa (TVP) y fibrilación ventricular (FV), de preferencia en hombres, adultos jóvenes, y durante el sueño nocturno ${ }^{4}$.

El patrón Br tipo I puede ser permanente o intermitente, apareciendo ante una estimulación vagal extrema, drogas antiarrítmicas del tipo IC, antidepresivos tricíclicos, trastornos electrolíticos, agentes anestésicos y, sobre todo, con el alza de la temperatura corporal ${ }^{2-4}$.

La anomalía de los canales del sodio más acentuada en la superficie del ventrículo derecho se expresaría a nivel de la fase 1 del potencial de acción celular y explicaría la anomalía tan característica del ECG, alterando la conducción y los períodos refractarios a nivel ventricular ${ }^{2,3}$.

La fiebre disminuye la activación y acelera la inactivación de los canales de sodio, los cuales $a$ priori están reducidos y disfuncionantes en los pacientes con $\mathrm{SBr}$, facilitando la aparición del

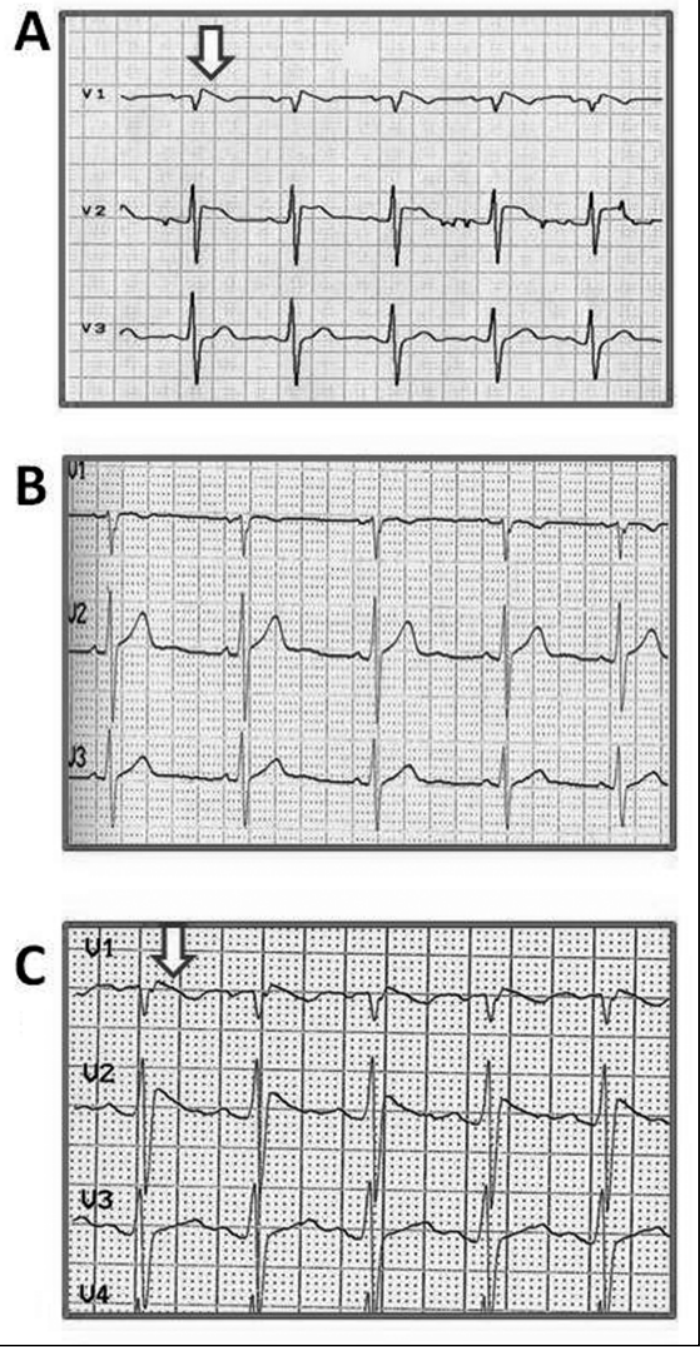

Figura 1. Derivaciones precordiales derechas (V1 a V3) del ECG registrado al ingreso durante el cuadro febril (A), a las 24 horas y sin fiebre (B) y con test de Procainamida ev (C). Nótese en $\mathrm{V} 1$ elevación descendente del segmento $\mathrm{ST} \geq 2 \mathrm{~mm}$ seguida de onda T negativa, patrón Brugada tipo I al ingreso y que se reproduce con Procainamida (flechas en A y C).

patrón Br tipo I con o sin la presencia de arritmias ventriculares malignas ${ }^{2,3,5}$.

Destacamos que el primer caso comunicado con $\mathrm{SBr}$ fue en un niño de 2 años que, durante un episodio febril por influenza, presentó múltiples episodios de TVP seguidos de colapso cardiocirculatorio $^{1}$. Posteriormente, en la literatura se han reportado algunos casos de $\mathrm{SBr}$ con el patrón $\mathrm{Br}$ tipo I asociados a influenza ${ }^{6} \mathrm{y}$ a otras enfermedades 
infecciosas como neumonía ${ }^{7}$, infección urinaria ${ }^{8}$ y shigellosis ${ }^{9}$, siendo el común denominador la presencia de fiebre. También se ha descrito en el curso de colecistitis y apendicitis aguda ${ }^{10}$.

La fiebre en el SBr es una combinación muy peligrosa, reportándose $17 \%$ de arritmias ventriculares malignas, $13 \%$ de paro cardíaco y $13 \%$ de síncope, por lo que es fundamental bajar rápidamente la temperatura con medidas físicas y farmacológicas ${ }^{10}$.

En nuestro medio planteamos que estos pacientes deben recibir las vacunas del programa nacional de inmunizaciones en la infancia y aquellas destinadas a pacientes adultos para evitar enfermedades infecciosas febriles, teniendo la precaución de usar una premedicación antipirética. En relación a la actual pandemia de COVID-19, se ha descrito que el virus puede provocar injuria miocárdica a través de distintos mecanismos fisiopatológicos, uno de los cuales podría ser por cardiotoxicidad directa, fundamentado en el reporte de casos clínicos aislados, observándose arritmias en $18 \%$ de los casos hospitalizados y en casi $50 \%$ de los pacientes admitidos en unidades intensivas, por lo que la monitorización del ECG es de gran importancia ${ }^{11}$. Cuando el intervalo QT es $\geq 500 \mathrm{~ms}$, se puede desencadenar una TVP por torsión de las puntas en aquellos pacientes que están recibiendo azitromicina o hidroxicloroquina aunque esta complicación no está asociadas al SBr ${ }^{11}$. Afortunadamente, las drogas empleadas en el tratamiento sintomático del COVID-19 no están contraindicadas en el $\mathrm{SBr}$, pero sí deben usarse cuidadosamente si existe un síndrome de QT largo asociado ${ }^{11}$.

La actual pandemia de COVID-19 plantea nuevos problemas de diagnóstico y tratamiento en pacientes que se presentan con fiebre, sospecha de infarto al miocardio (IAM) o aquellos recuperados de un síncope o MS. La fiebre puede desenmascarar un patrón Br tipo I al ECG y simular un IAM e inducir a un tratamiento inadecuado ${ }^{4,10}$.

La fiebre puede gatillar una taquiarritmia ventricular maligna ${ }^{5,10}$ en un paciente hasta ese momento asintomático. Recientemente se han reportado casos de infección febril por COVID-19 que han hecho evidente un $\mathrm{SBr}$ con patrón $\mathrm{Br}$ tipo $\mathrm{I}^{12,13}$.

Por todo lo anterior es que, en esta época de pandemia, recomendamos que todo paciente conocido por tener un patrón Br tipo I al ECG o un
$\mathrm{SBr}$ que cursa con un cuadro febril sospechoso o confirmado de COVID-19, debe consultar en un servicio de urgencia para su evaluación y manejo oportuno.

\section{Referencias}

1. Brugada P, Brugada J. Right Bundle branch block, persistent ST segment elevation and sudden cardiac death: a distinct clinical and electrocardiographic syndrome: A multicenter report. J Am Coll Cardiol 1992; 20: 1391-6.

2. Benito B, Brugada J, Brugada R, Brugada P. Síndrome de Brugada. Rev Esp Cardiol 2009; 62 (11): 1297-315.

3. Berne P, Brugada J. Brugada syndrome 2012. Circ J. 2012; 76: 1563-71.

4. Copolla G, Corrado E, Curnies A, Maglia G, Oriente D, et al. Update on Brugada Syndrome. Curr Prob Cardiol 2019; 00: 100454.

5. González-Rebollo JM, García A, Mejías A, Hernández-Madrid A, García de Castro A, Moro C. Fibrilación ventricular recurrente durante un proceso febril en un paciente con un síndrome de Brugada. Rev Esp Cardiol 2000; 53 (5): 755-7.

6. Baranchuk A, Simpson CS. Brugada syndrome coinciding with fever and pandemic (H1N1) influenza. CMAJ 2011; 183 (5): 852.

7. Sovari AA, Prasun MA, Kocheril AG, Brugada R. Brugada syndrome unmasked by pneumonia. Tex Heart Inst J 2006; 33 (4): 501-4.

8. Lamelas P, Labadet C, Spernanzoni F, López-Saubidet C, Alvarez PA. Brugada electrocardiographic pattern induced by fever. World J Cardiol 2012; 4: 84-6.

9. Makaryus JN, Verbsky J, Schwartz S, Slotwiner D. Fever associated with gastrointestinal shigellosis unmasks probable Brugada syndrome. Case Report Med 2009; 492031.

10. Roterberg G, El-Battrawy I, Veith M, Volker L, Ansari U, Lang S, et al. Arrhythmic events in Brugada syndrome patients induced by fever. Ann Noninvasive Electrocardiol 2020; 25: e12723. DOI: 10.1111/anec.12723.

11. Wu C, Postema P, Arbelo E, Behr ER, Bezzina CR, Napolitano C, et al. SARS-COVID-19 and inherited arrhytmia syndrome. Heart Rhytm 2020; pii: S15475271(20)30285-X.

12. Vidovich MI. Transient Brugada-like ECG pattern in a patient with Coronavirus Disease 2019 (COVID-19), JACC Case Reports (2020). doi: https://doi.org/10.1016/ j.jaccas.2020.04.007.

13. Chang D, Saleh M, Garcia-Bengo Y, Choi E, Epstein L, Willner J. COVID-19 Infection Unmasking Brugada Syndrome. Heart Rhythm Case Reports 2020. 